\title{
WEIGHTED NORM INEQUALITIES FOR OPERATORS OF HARDY TYPE
}

\author{
STEVEN BLOOM AND RON KERMAN
}

(Communicated by Andrew M. Bruckner)

\begin{abstract}
A new proof, yielding new conditions, is given for the two-weighted norm Hardy inequality. The theorem is extended to operators with kernels behaving much like the Riemann-Liouville fractional integrals of nonnegative order.
\end{abstract}

\section{HARDY'S INEQUALITY}

In this paper we present a new and easily proven criterion for the Hardy operator

$$
I f(x)=\int_{0}^{x} f(y) d y, \quad x>0
$$

to satisfy a weighted norm inequality. The criterion involves the adjoint operator

$$
I^{*} g(x)=\int_{x}^{\infty} g(y) d y .
$$

Using a more complicated version of the proof for I, we are able to obtain, in $\S I I$, similar criteria for weighted norm inequalities to hold for, among other operators, those of Riemann-Liouville,

$$
I_{\alpha} f(x)=\int_{0}^{x}(x-y)^{\alpha} f(y) d y, \quad x \text { and } \alpha>0 .
$$

These criteria are related to other, recently obtained, characterizations for $I_{\alpha}$ in $\S$ III.

The following special case of the result for I gives the essentials of our approach. The method of proof is similar to the classical proof of Hardy's inequality [1, p. 242-3].

Theorem 1.1. Fix $1<p<\infty$. Let $u$ and $v$ be nonnegative, measurable functions on $(0, \infty)$, with $0<u, v<\infty$ a.e. Then,

$$
\int_{0}^{\infty}(u I f)^{p} \leq C \int_{0}^{\infty}(v f)^{p}
$$

Received by the editors March 9, 1990.

1980 Mathematics Subject Classification (1985 Revision). Primary 26D15, 26A33.

The first author's research was supported in part by a grant from Siena College.

The second author's research was supported in part by NSERC grant A4021. 
for all nonnegative $f$, if and only if,

$$
I^{*}\left[\left(v^{-1} I^{*} u^{p}\right)^{p^{\prime}}\right] \leq C I^{*} u^{p}<\infty,
$$

where $p^{\prime}$ is the conjugate exponent $p /(p-1)$.

Proof. We may restrict our attention to bounded and nonnegative $f$ that are compactly supported in $(0, \infty)$. Then an integration by parts gives the identity

$$
\int_{0}^{\infty}(u I f)^{p}=p \int_{0}^{\infty} f(I f)^{p-1} I^{*} u^{p}
$$

This simple identity is the key to our proof. For if $\int_{0}^{\infty}(v f)^{p}=1$, then Hölder's inequality and another integration by parts gives

$$
\begin{aligned}
\int_{0}^{\infty}(u I f)^{p} & \leq p\left[\int_{0}^{\infty}(I f)^{p}\left(v^{-1} I^{*} u^{p}\right)^{p^{\prime}}\right]^{1 / p^{\prime}} \\
& =p\left[\int_{0}^{\infty} I^{*}\left[\left(v^{-1} I^{*} u^{p}\right)^{p^{\prime}}\right] d(I f)^{p}\right]^{1 / p^{\prime}} .
\end{aligned}
$$

Now using (1.2) and a further integration by parts, we obtain

$$
\int_{0}^{\infty}(u I f)^{p} \leq C\left[\int_{0}^{\infty} I^{*} u^{P} d(I f)^{p}\right]^{1 / p^{\prime}}=C\left[\int_{0}^{\infty}(u I f)^{p}\right]^{1 / p^{\prime}}<\infty
$$

and so,

$$
\int_{0}^{\infty}(u I f)^{p} \leq C .
$$

To see that (1.2) is implied by (1.1), observe that for any of our $f$ with $\int(v f)^{p}<\infty$, we have

$$
p \int_{0}^{\infty} f(I f)^{p-1} I^{*} u^{p}=\int_{0}^{\infty}(u I f)^{p} \leq C \int_{0}^{\infty}(v f)^{p}<\infty .
$$

This means that $I^{*} u^{p}<\infty$ on $(0, \infty)$. Substituting

$$
h(\cdot)=u(\cdot)^{p} \chi_{(x, \infty)}(\cdot)
$$

into the (equivalent) inequality

$$
\int_{0}^{\infty}\left(v^{-1} I^{*} h\right)^{p^{\prime}} \leq C \int\left(u^{-1} h\right)^{p^{\prime}}
$$

dual to (1.1), we obtain (1.2).

\section{RIEMANN-LIOUVILLE OPERATORS}

It will be convenient to prove our result for kernels somewhat more general than those of Riemann-Liouville. We consider kernels $\varphi(x, y)$ on $\mathbb{R}^{+} \times \mathbb{R}^{+}$ with the following properties:

(i) $\varphi(x, y)>0$ if $x>y$;

(ii) $\varphi(x, y)$ is nondecreasing in $x$ and nonincreasing in $y$;

(iii) $\varphi(x, y) \approx \varphi(x, z)+\varphi(z, y)$ if $y<z<x$. 
Given $r \geq 0$, we set

$$
T_{r} f(x)=\int_{0}^{x} \varphi(x, y)^{r} f(y) d y
$$

and

$$
T_{r}^{*} g(x)=\int_{x}^{\infty} \varphi(y, x)^{r} g(y) d y .
$$

We will write $T$ for $T_{1}$ and $I$ for $T_{0}$.

We can now state our principal result.

Theorem 2.1. Fix $1<p \leq q<\infty$. Let $u$ and $v$ be nonnegative, measurable functions on $(0, \infty)$ with $0<u, v<\infty$ a.e. Then,

$$
\left[\int_{0}^{\infty}(u T f)^{q}\right]^{1 / q} \leq C\left[\int_{0}^{\infty}(v f)^{p}\right]^{1 / p} \text { for all } f \geq 0
$$

if and only if

$$
I^{*}\left[\left(v^{-1} T^{*} u^{q}\right)^{p^{\prime}}\right] \leq C\left(I^{*} u^{q}\right)^{p^{\prime} / q^{\prime}}<\infty
$$

and

$$
I^{*}\left[\left(v^{-1} T_{q}^{*} u^{q}\right)^{p^{\prime}}\right] \leq C\left(T_{q}^{*} u^{q}\right)^{p^{\prime} / q^{\prime}}<\infty
$$

a.e. on $(0, \infty)$.

To prove this, we want an analog of the elementary identity (1.3) used in the proof of Theorem 1.1. This is given in the following lemma.

Lemma 2.2. Fix $1<p<\infty$. Then for all $f$ and $u \geq 0$ with $\int(u T f)^{p}<\infty$,

$$
\int_{0}^{\infty}(u T f)^{p} \approx \int_{0}^{\infty} f(I f)^{p-1} T_{p}^{*} u^{p}+\int_{0}^{\infty} f(T f)^{p-1} T^{*} u^{p} .
$$

Observe that when $T$ is the Hardy operator $I$, so is each $T_{r}$, and both terms on the right side of $(2.5)$ are equal to $p^{-1}$ times the right side of (1.3).

Proof of the lemma. Given $f$ not identically zero, there is a greatest $x_{0} \geq 0$ with $f=0$ a.e. on $\left[0, x_{0}\right]$, so that $I f(x)$ and $T f(x)$ are positive when $x>x_{0}$. Taking adjoints,

$$
\int_{0}^{\infty}(u T f)^{p}=\int_{x_{0}}^{\infty} f T^{*}[w T f]
$$

where $w=u^{p}(T f)^{p-2}$. Again, when $x>x_{0}$,

$$
\begin{aligned}
T^{*}[w T f](x)= & \int_{x}^{\infty} \varphi(y, x) w(y) d y\left(\int_{0}^{x}+\int_{x}^{y}\right) \varphi(y, z) f(z) d z \\
= & \int_{x}^{\infty} \varphi(y, x) w(y) d y \int_{0}^{x} \varphi(y, z) f(z) d z \\
& +\int_{x}^{\infty} f(z) d z \int_{z}^{\infty} \varphi(y, x) \varphi(y, z) w(y) d y \\
= & A(x)+B(x) .
\end{aligned}
$$


Now, by $(2.1)($ iii),

$$
\begin{aligned}
& A \approx(I f)\left(T_{2}^{*} w\right)+(T f)\left(T^{*} w\right), \\
& B \approx I^{*}\left[f I_{2}^{*} w\right]+T^{*}\left[f T^{*} w\right] .
\end{aligned}
$$

Fubini's theorem and the equivalences (2.7) and (2.6) show

$$
\int_{0}^{\infty}(u T f)^{p} \approx \int_{x_{0}}^{\infty} f(I f) T_{2}^{*} w+\int_{x_{0}}^{\infty} f(T f) T^{*} w .
$$

This proves the lemma when $p=2$. Suppose then that $p>2$. Hölder's inequality with exponents $p-1$ and $(p-1) /(p-2)$ yields

$$
T_{2}^{*} w \leq\left[T^{*}\left(u^{p}(T f)^{p-1}\right]^{(p-2) /(p-1)}\left[T_{p}^{*} u^{p}\right]^{1 /(p-1)}\right.
$$

and

$$
T^{*} w \leq\left[T^{*}\left(u^{p}(T f)^{p-1}\right]^{(p-2) /(p-1)}\left[T^{*} u^{p}\right]^{1 /(p-1)} .\right.
$$

Hence, by another use of Hölder's inequality with the same exponents,

$$
\begin{aligned}
& \int_{x_{0}}^{\infty} f(I f) T_{2}^{*} w \leq\left[\int_{x_{0}}^{\infty} f(I f)^{p-1} T_{p}^{*} u^{p}\right]^{1 /(p-1)}\left[\int_{x_{0}}^{\infty}(u T f)^{p}\right]^{(p-2) /(p-1)} \\
& \int_{x_{0}}^{\infty} f(I f) T^{*} w \leq\left[\int_{x_{0}}^{\infty} f(T f)^{p-1} T^{*} u^{p}\right]^{1 /(p-1)}\left[\int_{x_{0}}^{\infty}(u T f)^{p}\right]^{(p-2) /(p-1)} .
\end{aligned}
$$

Using the estimates (2.9) and (2.8) yields, after some simplification, (2.5), but as an inequality, rather than an equivalence.

Next, suppose $1<p<2$. For $x>x_{0}$,

$$
\begin{aligned}
T_{2}^{*} w(x) & =\int_{x}^{\infty} \varphi(y, x)^{2} u(y)^{p} T f(y)^{p-2} d y \\
& =\int_{x}^{\infty} \varphi(y, x)^{p} u(y)^{p}[T f(y) / \varphi(y, x)]^{p-2} d y \\
& \leq I f(x)^{p-2} T_{p}^{*} u^{p}(x)
\end{aligned}
$$

by $(2.1)(\mathrm{ii})$, and

$$
\begin{aligned}
T^{*} w(x) & =\int_{x}^{\infty} \varphi(y, x) u(y)^{p} T f(y)^{p-2} d y \\
& \leq T f(x)^{p-2} T^{*} u^{p}(x) .
\end{aligned}
$$

Substituting these estimates in (2.8) gives one of the inequalities implicit in (2.5).

For the opposite inequality, when $p>2$,

$$
T_{r}^{*} w(x) \geq \int_{x}^{\infty} \varphi(y, x)^{r} u(y)^{p} d y\left(\int_{0}^{x} \varphi(y, z) f(z) d z\right)^{p-2} .
$$

Thus, by $(2.1)(\mathrm{ii})$,

$$
T_{2}^{*} w(x) \geq I f(x)^{p-2} T_{p}^{*} u^{p}(x)
$$


and

$$
T^{*} w(x) \geq T f(x)^{p-2} T^{*} u^{p}(x) .
$$

These, coupled with (2.9), prove (2.5) when $p>2$.

When $p<2$, we appeal again to Hölder's inequality, this time with exponents $1 /(p-1)$ and $1 /(2-p)$.

$$
\begin{aligned}
T_{p}^{*} u^{p}(x) & =\int_{x}^{\infty} \varphi(y, x) u^{p}(y) \varphi(y, x)^{p-1} T f(y)^{(p-2)(p-1)} T f(y)^{(2-p)(p-1)} d y \\
& \leq\left[T_{2}^{*} w\right]^{p-1}\left[T^{*}\left(u^{p}(T f)^{p-1}\right)\right]^{2-p}
\end{aligned}
$$

and so, by another application of Hölder's inequality,

$$
\begin{aligned}
\int f(I f)^{p-1} T_{p}^{*} u^{p} & \leq\left[\int f(I f) T_{2}^{*} w\right]^{p-1}\left[\int f T^{*}\left(u^{p}(T f)^{p-1}\right)\right]^{2-p} \\
& =\left[\int f(I f) T_{2}^{*} w\right]^{p-1}\left[\int(u T f)^{p}\right]^{2-p} .
\end{aligned}
$$

Similarly,

$$
T^{*} u^{p} \leq\left[T^{*} w\right]^{p-1}\left[T^{*} u^{p}(T f)^{p-1}\right]^{2-p}
$$

and

$$
\int f(T f)^{p-1} T^{*} u^{p} \leq\left[\int f(T f) T^{*} w\right]^{p-1}\left[\int(u T f)^{p}\right]^{2-p},
$$

which, in view of the equivalence (2.8), proves (2.5).

Proof of Theorem 2.1. In proving the only if part of the theorem, we appeal to the (equivalent) inequality

$$
\left[\int_{0}^{\infty}\left(v^{-1} T^{*} g\right)^{p^{\prime}}\right]^{1 / p^{\prime}} \leq C\left[\int_{0}^{\infty}\left(u^{-1} g\right)^{q^{\prime}}\right]^{1 / q^{\prime}}
$$

dual to (2.2).

Suppose (2.2) holds. Given any $f>0$ with $\int(u f)^{p}<\infty$, we have, by (2.2) and $(2.5)$,

$$
\int_{0}^{\infty} f(I f)^{q-1} T_{q}^{*} u^{q} \leq C \int_{0}^{\infty}(u T f)^{q} \leq C\left[\int_{0}^{\infty}(v f)^{q}\right]^{q / p}<\infty
$$

from which we conclude that $T_{q}^{*} u^{q}<\infty$ on $(0, \infty)$. Moreover, from (2.1)(ii),

$$
T_{q}^{*} u^{q}(x / 2) \geq \varphi(x, x / 2)^{q}\left(I^{*} u^{q}\right)(x)
$$

and so $I^{*} u^{q}$ is also finite. Now, for $r \geq 1$,

$$
\begin{gathered}
I^{*}\left[\left(v^{-1} T_{r}^{*} u^{q}\right)^{p^{\prime}}\right](x)=\int_{x}^{\infty} v(y)^{-p^{\prime}} d y\left[\int_{y}^{\infty} \varphi(z, y)^{r} u(z)^{q} \chi_{(x, \infty)}(z) d z\right]^{p^{\prime}} \\
\leq \int_{0}^{\infty} v(y)^{-p^{\prime}} d y\left[\int_{y}^{\infty} \varphi(z, y) \varphi(z, x)^{r-1} u(z)^{q} \chi_{(x, \infty)}(z) d z\right]^{p^{\prime}}
\end{gathered}
$$


by $(2.1)(\mathrm{ii})$,

$$
\leq C\left[\int_{x}^{\infty} \varphi(z, x)^{(r-1) q^{\prime}} u(z)^{q} d z\right]^{p^{\prime} / q^{\prime}}
$$

by (2.10). This estimate is (2.3) when $r=1$ and gives (2.4) when $r=q$.

In the converse direction, we assume (2.3) and (2.4) and argue from (2.5) much as in the proof of Theorem 1.1. We will show that the integral $\int_{0}^{\infty}(u T f)^{q}$ is bounded independently of bounded, nonnegative,compactly supported $f$ on $(0, \infty)$ with $\int(v f)^{p}=1$. It is clearly finite.

Now let $(r, s)=(0, q)$ or $(1,1)$ and let $t=(s-1) q^{\prime}$. Then Hölder's inequality and an integration by parts gives

$$
\begin{aligned}
\int_{0}^{\infty} f\left(T_{r} f\right)^{q-1} T_{s}^{*} u^{q} & \leq\left[\int_{0}^{\infty}(v f)^{p}\right]^{1 / p}\left[\int_{0}^{\infty}\left(T_{r} f\right)^{p^{\prime}(q-1)}\left(v^{-1} T_{s}^{*} u^{q}\right)^{p^{\prime}}\right]^{1 / p^{\prime}} \\
& =\left[\int_{0}^{\infty} I^{*}\left[\left(v^{-1} T_{s}^{*} u^{q}\right)^{p^{\prime}}\right] d\left(T_{r} f\right)^{p^{\prime}(q-1)}\right]^{1 / p^{\prime}}
\end{aligned}
$$

which by assumption, is bounded by a constant times

$$
\begin{aligned}
J & =\left[\int_{0}^{\infty}\left(T_{t}^{*} u^{q}\right)^{p^{\prime} / q^{\prime}} d\left(T_{r} f\right)^{p^{\prime}(q-1)}\right]^{1 / p^{\prime}} \\
& =\left[\int_{0}^{\infty}\left(I^{*}\left[-d T_{t}^{*} u^{q}\right]\right)^{p^{\prime} / q^{\prime}} d\left(T_{r} f\right)^{p^{\prime}(q-1)}\right]^{1 / p^{\prime}} .
\end{aligned}
$$

By Minkowski’s inequality,

$$
J \leq\left[\int_{0}^{\infty}\left(-d T_{t}^{*} u^{q}\right)\left(T_{r} f\right)^{q}\right]^{1 / q^{\prime}}
$$

and another integration by parts yields

$$
J \leq \begin{cases}{\left[\int f(I f)^{q-1} T_{q}^{*} u^{q}\right]^{1 / q^{\prime}}} & \text { when }(r, s)=(0,1), \\ {\left[\int(u T f)^{q}\right]^{1 / q^{\prime}}} & \text { when }(r, s)=(1,1)\end{cases}
$$

which, in view of (2.5), concludes the proof.

\section{OTHER CRITERIA}

Stepanov [3] proves that the following conditions are necessary and sufficient for $T=I_{\alpha}$ to satisfy (2.2).

$$
\left(I_{\alpha p^{\prime}} v^{-p^{\prime}}\right)^{1 / p^{\prime}}\left(I^{*} u^{q}\right)^{1 / q} \leq C<\infty
$$

and

$$
\left(I v^{-p^{\prime}}\right)^{1 / p^{\prime}}\left(I_{\alpha q}^{*} u^{q}\right)^{1 / q} \leq C<\infty
$$


Using different methods, Martin-Reyes and Sawyer [2] obtain similar conditions for a more general class of operators. In this section, we show that conditions (3.1) and (3.2) imply (2.3) and (2.4) respectively, with $T_{r}=I_{\alpha r}$; that is

$$
I^{*}\left[\left(v^{-1} I_{\alpha}^{*} u^{q}\right)^{p^{\prime}}\right] \leq C\left(I^{*} u^{q}\right)^{p^{\prime} / q^{\prime}}<\infty
$$

and

$$
I^{*}\left[\left(v^{-1} I_{\alpha q}^{*} u^{q}\right)^{p^{\prime}}\right] \leq C\left(I_{\alpha q}^{*} u^{q}\right)^{p^{\prime} / q^{\prime}}<\infty .
$$

The proof that (3.1) implies (3.3) is essentially contained in Stepanov's work. To see that (3.2) implies (3.4), assume first that $\left(I_{\alpha q}^{*} u^{q}\right)\left(0^{+}\right)=\infty$. Then $\left(I_{\alpha q}^{*} u^{q}\right)^{-p^{\prime} / q}=I g$ for some $g \geq 0$. Define $v_{0}$ by $v_{0}^{-p^{\prime}}=v^{-p^{\prime}}+g$, so $I v_{0}^{-p^{\prime}} \approx\left(I_{\alpha q}^{*} u^{q}\right)^{-p^{\prime} / q}$. We have

$$
\begin{aligned}
I^{*}\left[\left(v^{-1} I_{\alpha q}^{*} u^{q}\right)^{p^{\prime}}\right] & \leq C I^{*}\left[\left(v_{0}^{-1} I_{\alpha q}^{*} u^{q}\right)^{p^{\prime}}\right] \\
& \leq C I^{*}\left[\left(v_{0}^{-1} I v^{-p^{\prime}}\right)^{-q}\right] \\
& \leq C\left(I v_{0}^{-p^{\prime}}\right)^{1-q} \\
& \leq C\left(I_{\alpha q}^{*} u^{q}\right)^{p^{\prime} / q^{\prime}} .
\end{aligned}
$$

Suppose next that $I_{\alpha q}^{*} u^{q}\left(0^{+}\right)<\infty$. If also $\int_{0}^{\infty} v^{-p^{\prime}}<\infty$, we are done. Otherwise, define $u_{0}$ by $\int_{x}^{\infty} y^{\alpha q} u_{0}(y)^{q} d y=\left[\int_{0}^{x} v(y)^{-p^{\prime}} d y\right]^{-q / p^{\prime}}$ and set $u_{\varepsilon}=u+\varepsilon u_{0}$. Then $u_{\varepsilon}$ and $v$ satisfy (3.2) and $I_{\alpha q}^{*} u_{\varepsilon}^{q}\left(0^{+}\right)=\infty$, so

$$
\left.I^{*}\left[v^{-1} I_{\alpha q}^{*} u_{\varepsilon}^{q}\right)^{p^{\prime}}\right] \leq C\left(I_{\alpha q}^{*} u_{\varepsilon}^{q}\right)^{p^{\prime} / q^{\prime}}<\infty .
$$

Letting $\varepsilon \rightarrow 0^{+}$gives (3.4).

\section{REFERENCES}

1. G. Hardy, J. Littlewood, and G. Polya, Inequalities, Cambridge Univ. Press, 1967.

2. F. Martin-Reyes and E. Sawyer, Weighted norm inequalities for the Riemann-Liouville fractional integral operators, preprint.

3. V. Stepanov, Two-weighted estimates for Riemann-Liouville integrals, preprint.

Department of Mathematics, Siena College, Loudonville, New York 12211

Department of Mathematics, Brock University, St. Catharines, Ontario, L2S 3A1 Canada 